\title{
MORUS - Utopia e Renascimento, Carlos Berriel editor
}

\section{Monia Mezzetti}

\section{(2) OpenEdition}

\section{Journals}

\section{Edizione digitale}

URL: http://journals.openedition.org/studifrancesi/423

DOI: 10.4000/studifrancesi.423

ISSN: 2421-5856

\section{Editore}

Rosenberg \& Sellier

\section{Edizione cartacea}

Data di pubblicazione: 1 aprile 2015

Paginazione: 139-140

ISSN: 0039-2944

\section{Notizia bibliografica digitale}

Monia Mezzetti, «MORUS - Utopia e Renascimento, Carlos Berriel editor», Studi Francesi [Online], 175

(LIX | I) | 2015, online dal 01 avril 2015, consultato il 18 septembre 2020. URL : http://

journals.openedition.org/studifrancesi/423 ; DOI : https://doi.org/10.4000/studifrancesi.423

\section{Questo documento è stato generato automaticamente il 18 settembre 2020.}

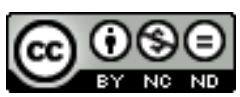

Studi Francesi è distribuita con Licenza Creative Commons Attribuzione - Non commerciale - Non opere derivate 4.0 Internazionale. 


\title{
MORUS - Utopia e Renascimento, Carlos Berriel editor
}

\author{
Monia Mezzetti
}

\section{NOTIZIA}

MORUS - Utopia e Renascimento, Carlos BERRIEL editor, Campinas (Brasil), n. 8, 2012 (Atti del III Congresso Internacional de Estudos Utópicos «Utopia, consenso e livre-arbítrio séculos XIV-XVII», Chambord-Tours, 26-27 gennaio 2012), pp. 245.

1 Questo ottavo volume del periodico brasiliano MORUS - Utopia e Renascimento risulta assolutamente stimolante per la scelta del tema, quello del concetto di libero arbitrio nelle alterità utopiche. Questo, infatti, di primo acchito parrebbe quanto di più estraneo al modus vivendi in utopia, improntato com'è al rispetto acritico di leggi ferree, a loro volta incompatibili con qualsivoglia forma di contributo individuale e semmai tributarie della volontà del fondatore dell'alterità stessa. Di questa rigidità normativa $\mathrm{e}$ del supino attenervisi da parte della popolazione utopiana non vi sarebbe in realtà nulla di sorprendente, se non fosse per la seconda imprescindibile peculiarità del mundus alter (dopo quella dell'inesistenza implicata dal termine $u$-topos), ovvero la sua positività: questa si trova infatti adombrata nel neologismo stesso di Thomas More $(u-$ topia $=$ eu-topia $)$. Eppure l'utopia non è mai stata il regno della libertà, della discrezionalità. Le rare eccezioni, in tal senso, sono rappresentate dal Paese di Cuccagna (della cui trattazione negli studi utopici Hilário FRANCISCO jr. (pp. 149-162) rivendica polemicamente la pertinenza in base a un criterio tematico, che mette in secondo piano quello temporale, implicato dalla distanza rispetto al modello moreano) e da quella singolare realtà che è l'abbazia di Thélème (tributaria, sempre secondo Hilário Francisco jr., del tema di ascendenza medievale che, sorprendentemente, scopriamo essere stato vitale fino a tutto il xvi secolo). Una sintesi della discussione, tenutasi a Chambord nel gennaio del 2012, sull'ermeneutica del celebre imperativo «Fais ce que voudras» è offerta alle pp. 19-26, con gli interventi di Nicolas LE CADET, 
Raphaël CAPPELLAN, Mireille HUCHON, Jean CÉARD. Di quest'ultimo è anche il primo contributo (pp. 27-40), dedicato alla ricezione del testo moreano in Francia nel '500 attraverso la lettura di Guillaume Budé e Du Verdier, nonché le traduzioni a cura di Le Blond e Barthélemy Aneau. Di Marie-Claire PHÉLIPPEAU (pp. 41-52) è invece il successivo saggio dal titolo Les portes étroites d'Utopia che esplora, sempre nell'opera di More, la percezione della pulsionalità, la sua legittimità, con esiti complessivamente negativi in ragione del vigente orientamento di stampo paolino, secondo cui l'individuo è essenzialmente un peccatore. Di Barthélemy Aneau torna ad occuparsi Yvonne GREIS (pp. 53-64), autrice di una traduzione in portoghese dell'Alector (1560), la quale mette in evidenza analogie e differenze tra le città di Amauroto e di Orba (cap. 24) sia da un punto di vista strutturale che sostanziale in relazione all'esercizio del libero arbitrio da parte dei rispettivi abitanti. Sempre all'opera di Rabelais è rivolto l'articolo di MarieLuce DEMONET, Utopie et dystopies chez Rabelais de "Pantagruel" au "Quart Livre" (pp. 105-118), che si sofferma sul binomio utopia/distopia così come appare nel regno dell'isola des Alliances (Quart Livre, cap. IX). All'ambito teatrale è riservato poi quello di Alessandra PREDA (pp. 119-130) che esamina questione editoriale e contenuto della tragedia in prosa di Francesco Negri, pubblicata, oltre che in Italia (1546), a Ginevra con il titolo Tragedie du Roy Franc-Arbitre (1558) e dove, nel solco della controversia religiosa, l'utopia è rappresentata dal mondo futuro, improntato agli insegnamenti del Vangelo, mentre il presente ha connotati distopici (corruzione del clero, superstizione...). I restanti contributi vertono invece sulla produzione secentesca, in particolare sui seguenti autori e testi: (a) La Città del Sole di Campanella (Germana ERNST, Comunità e libertà in C. (pp. 175-191); Carlos BERRIEL, La soppressione della peccaminosità del mondo (pp. 192-202); Jean-Louis FOURNEL, Y a-t-il des terres inconnues? Considérations sur l'utopie selon C. (pp. 203-216 ); (b) Il labirinto del mondo di Comenio (Iveta NAKLÁDALovÁ, pp. 65-76); (c) The Isle of Pines di Henry Neville (Helvio MORAES, pp. 77-85) (Segnaliamo una peculiarità del testo (per l'esattezza un pamphlet), ovvero l'allestimento attraverso un technical device che sarà caro agli utopisti francesi dell'Ottocento (Le Hon, Franklin, Jules e/o Michel Verne, Le dernier Adam), quello della redazione di una sorta di diario, ad uso dei posteri, da parte dei superstiti di una catastrofe naturale vissuti isolatamente in qualche punto remoto del pianeta); (d) la Terre Australe di Gabriel de Foigny (Ana Cláudia Romano RIBEIRO, La "Genèse" réécrite, pp. 86-95); (e) la Reipublicae Christianopolitanae di Andreä (Maurizio CAMBI, pp. 96-104); (f) Mondo pazzo, mondo savio di Anton Francesco Doni e La Città felice di Patrizi (Gianluca BonAIUTI, Ritratto dell'utopico politico italiano, pp. 131-148); (g) Evandria di Lodovico Zuccolo (Claudio DE BONI, Fra ragion di Stato e nostalgia repubblicana, pp. 217-229); (h) i Dialoghi di Antonio Brucioli (Chiara LASTRAIOLI, Utopies célèstres et terrestres dans la production d'A.B., pp. 231-245). (i) In antitesi, le Pensées di Pascal (Hélène michon, P. et le rejet de la cité de Dieu, pp. 163-174). Ciascun saggio è corredato dalla bibliografia effettivamente utilizzata. Il volume risulta complessivamente ben bilanciato e costituito da contributi di sicuro interesse per l'approfondimento di un tema, quale quello del libero arbitrio, che della modalità speculativa utopica rappresenta sia il tratto distintivo sia il punto debole, in quanto possibile incipit di un'involuzione verso la distopia (sull'argomento, cfr. Utopia. Storia e teoria di un'esperienza filosofica e politica, a cura di Carlo Altini, Bologna, Il Mulino, 2013, pp. 542 ISBN: 978-88-15-245114-4 - nostra recensione in www.universomondo.it (in corso di pubblicazione). 